\title{
On the number of droplets in aerosols
}

\author{
H. Gouin ${ }^{\mathrm{a} *}$, F. Cubisol ${ }^{\mathrm{a}}$ \\ a'Université d'Aix-Marseille, Lab. Modélisation en Mécanique et Thermodynamique, \\ Ave Escadrille Normandie-Niemen, 13397 Marseille Cedex 20, France
}

The number of droplets which may be formed with a supersaturated vapor in presence of a gas cannot exceed a number proportional to $\left(p_{v}-p_{v_{0}}\right)^{4}$ where $p_{v}$ and $p_{v_{0}}$ denote at the same temperature the pressure of the supersaturated vapor-gas mixture and the pressure of the saturated vapor-gas mixture. The energy necessary to the droplet formation is also bounded by a number proportional to $\left(p_{v}-p_{v_{0}}\right)^{2}$.

Keywords: aerosols, droplets, liquid-vapor mixture.

\section{INTRODUCTION}

Aerosols are usual in industries of cosmetics, pharmacy and food. One also finds them in problems involving environment and pollution. Epidemics, radioactive elements can be carried by small droplets; volcanoes generate sulphated aerosols contributing to the destruction of the ozone layer. The planetary atmospheres are charged with such mixtures in suspension. The interaction between oceans and atmosphere generates mixtures of gas and water vapor partially condensed in form of fine droplets. Aerosols are also created by sprays and are important even in the car industry on the level of combustion in petrol and air mixture. The literature abounds on the study of nucleation times, on the growth of germs in vapor mixtures and on the motion of microscopic droplets [ 1, 2].

Aerosols consist of liquid droplets of very small radius (from 0.1 to 10 microns) in suspension in a vapor-gas mixture; the droplet number is assumed weak enough to avoid interactions (smaller than one hundred thousand droplets per a cubic centimeter which corresponds to a volume proportion smaller than one for thousand).

In this paper, we prove that for given conditions of pressure and temperature the number of droplets per unit volume cannot exceed a maximum value. The value depends on the surface tension of gas-vapor/liquid interface and on the difference between the pressure of gas supersaturated with vapor and that of gas with saturated vapor. The energy necessary to the creation of the droplets is limited; providing to a gas-vapor mixture an energy higher than this limit does not increase the droplet density.

*e-mail: henri.gouin@univ-cezanne.fr 


\section{POSITION OF THE PROBLEM}

In a large-sized isothermal tank of volume $V$, we consider a mixture constituted of two fluids labelled with indices 1 and 2. Fluid 1 is supposed at a temperature much lower than its critical temperature and coexists in form of liquid and vapor bulks. Fluid 2 is supposed at a temperature much upper than its critical temperature and behaves like a perfect gas (we call it the gas). An example of mixture verifying such conditions is that of water in liquid and vapor forms in presence of air at the ambient temperature. We propose to determine the equilibrium of $n$ liquid droplets with a dissolved gas in presence of supersaturated vapor in the gas. The droplets form a fog and their volume fraction is small enough so that they do not interact. The tank is large enough so that the boundary effects are neglected. Let us denote $\rho_{i m} V$ the total mass of fluid $i(i=1,2)$. The mixture is distributed between a gas-vapor bulk $v$ of volume $v_{v}$ and whose densities are denoted $\rho_{i v}$ and the $n$ droplets of liquid with dissolved gas of densities $\rho_{i l}$ represented by a bulk $l$. The radius droplet is small (of micron order) and the forces due to gravity are negligible. At equilibrium, the droplets are spherical and it is easy to notice that they are all identical with a volume $v_{l}$. The volume free energy of the mixture is denoted $\Psi\left(\rho_{1}, \rho_{2}\right)$. In a van der Waals model, it is a non convex function of the densities $\rho_{1}$ and $\rho_{2}$ [3]. The surface tension $\sigma$ of interfaces separating the bulks $l$ and $v$ is assumed to be independent of $\rho_{1}$ and $\rho_{2}$. This approximation corresponds to the fact that density values will be limited to small domains near the phase equilibrium. By taking into account the surface energy of the $n$ droplets, the total free energy of the mixture in the tank is

$E=n \Psi\left(\rho_{1 l}, \rho_{2 l}\right) v_{l}+\Psi\left(\rho_{1 v}, \rho_{2 v}\right) v_{v}+\frac{3}{2} \frac{n}{K^{\frac{1}{3}}} v_{l}^{\frac{2}{3}}$

with $K=\frac{3}{32 \pi \sigma^{3}}$. Volumes and densities of the mixture bulks are submitted to the constraints

$\left\{\begin{array}{l}n v_{l}+v_{v}=V \\ n \rho_{1 l} v_{l}+\rho_{1 v} v_{v}=\rho_{1 m} V \\ n \rho_{2 l} v_{l}+\rho_{2 v} v_{v}=\rho_{2 m} V\end{array}\right.$

The research of mixture equilibrium reduces to study the extremum of

$F=E-\lambda\left(n v_{l}+v_{v}\right)-\mu\left(n \rho_{1 l} v_{l}+\rho_{1 v} v_{v}\right)-\nu\left(n \rho_{2 l} v_{l}+\rho_{2 v} v_{v}\right)$

Where $\lambda, \mu, \nu$ are three scalar Lagrange multipliers. The balance equations associated with the variations of $v_{l}, v_{v}, \rho_{1 l}, \rho_{2 l}, \rho_{1 v}, \rho_{2 v}$ are

$$
\begin{cases}n \Psi\left(\rho_{1 l}, \rho_{2 l}\right)+n\left(K v_{l}\right)^{-\frac{1}{3}}-n \lambda-n \mu \rho_{1 l}-n \nu \rho_{2 l} & =0 \\ \Psi\left(\rho_{1 v}, \rho_{2 v}\right)-\lambda-\mu \rho_{1 v}-\nu \rho_{2 v} & =0 \\ n \Psi_{\rho_{1}}^{\prime}\left(\rho_{1 l}, \rho_{2 l}\right) v_{l}-\mu n v_{l} & =0 \\ n \Psi_{\rho_{2}}^{\prime}\left(\rho_{1 l}, \rho_{2 l}\right) v_{l}-\nu n v_{l} & =0 \\ \Psi_{\rho_{1}}^{\prime}\left(\rho_{1 v}, \rho_{2 v}\right) v_{v}-\mu v_{v} & =0 \\ \Psi_{\rho_{2}}^{\prime}\left(\rho_{1 v}, \rho_{2 v}\right) v_{v}-\nu v_{v} & =0\end{cases}
$$

For the sake of simplicity, we denote also

$$
\psi(l)=\Psi\left(\rho_{1 l}, \rho_{2 l}\right), \psi(v)=\Psi\left(\rho_{1 v}, \rho_{2 v}\right), \psi_{i}^{\prime}(l)=\Psi_{\rho_{i}}^{\prime}\left(\rho_{1 l}, \rho_{2 l}\right), \psi_{i}^{\prime}(v)=\Psi_{\rho_{i}}^{\prime}\left(\rho_{1 v}, \rho_{2 v}\right)
$$


with $i=1,2$. One deduces the relations of equilibrium

$\psi_{1}^{\prime}(l)=\psi_{1}^{\prime}(v)=\mu$

$\psi_{2}^{\prime}(l)=\psi_{2}^{\prime}(v)=\nu$

$\psi(v)-\rho_{1 v} \psi_{1}^{\prime}(v)-\rho_{2 v} \psi_{2}^{\prime}(v)-\psi(l)+\rho_{1 l} \psi_{1}^{\prime}(l)+\rho_{2 l} \psi_{2}^{\prime}(l)=\left(\frac{1}{K v_{l}}\right)^{\frac{1}{3}}$

and the lagrange multiplier $\lambda=\psi(v)-\rho_{1 v} \psi_{1}^{\prime}(v)-\rho_{2 v} \psi_{2}^{\prime}(v)$. Relations (5) and (6) express the equality of the chemical potentials of each mixture component in the two bulks $l$ and $v$ (see Fig. 1). At the temperature $T_{0}$, the total pressure value is

$p\left(\rho_{1}, \rho_{2}\right)=\rho_{1} \Psi_{\rho_{1}}^{\prime}\left(\rho_{1}, \rho_{2}\right)+\rho_{2} \Psi_{\rho_{2}}^{\prime}\left(\rho_{1}, \rho_{2}\right)-\Psi\left(\rho_{1}, \rho_{2}\right)+p_{o}$

where $p_{0}$ is a reference pressure and relation (7) is equivalent to

$p_{l}-p_{v}=\frac{2 \sigma}{R}$

where $R$ is the radius of the droplets and $p_{l}, p_{v}$ denote the pressure in bulks $l$ and $v$. Relation (8) is the expression of Laplace equation for spherical interfaces of fluid mixtures [4].

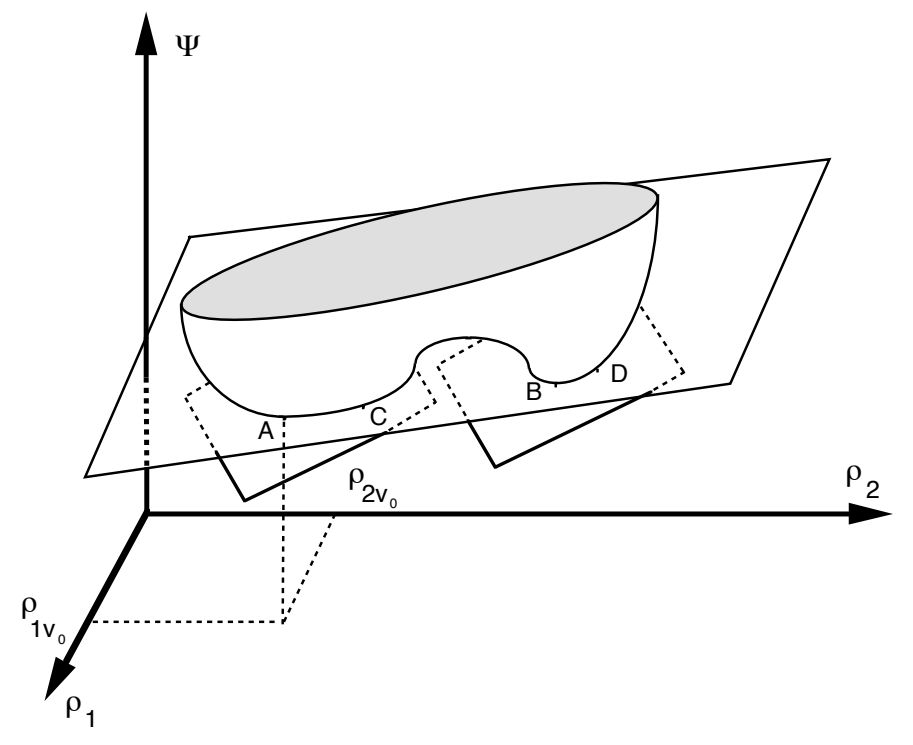

Figure 1. For a plane interface, the balance of phases is associated with contact points A and $\mathrm{B}$ of a bi-tangential plan to the free energy surface. Points C and D correspond to the balance of phases between a phase corresponding to droplets and the gas-vapor bulk. Tangent plans at points $\mathrm{C}$ and $\mathrm{D}$ to the free energy surface are parallel [ 5 . 


\section{THE DROPLET NUMBER PER UNIT VOLUME OF THE MIXTURE}

Relations (2) make it possible to write:

$v_{l}=\frac{\rho_{i m}-\rho_{i v}}{n\left(\rho_{i l}-\rho_{i v}\right)} V \quad v_{v}=\frac{\rho_{i l}-\rho_{i m}}{\rho_{i l}-\rho_{i v}} V \quad(i=1,2)$

Expression (7) yields

$N=K \frac{\rho_{i m}-\rho_{i v}}{\rho_{i l}-\rho_{i v}}\left[p\left(\rho_{1 l}, \rho_{2 l}\right)-p\left(\rho_{1 v}, \rho_{2 v}\right)\right]^{3}$

where $N=n / V$ is the number of droplets per unit volume of the mixture. Let us notice that $0 \leq \frac{\rho_{i m}-\rho_{i v}}{\rho_{i l}-\rho_{i v}} \leq 1$. For $\rho_{i v}=\rho_{i v_{o}}$ then $\rho_{i l}=\rho_{i l_{o}}$ where $\rho_{i v_{o}}, \rho_{i l_{o}}$ are the densities in the bulks of component $i$ for a liquid-vapor plane interface, we obtain $p\left(\rho_{1 l_{o}}, \rho_{2 l_{o}}\right)=$ $p\left(\rho_{1 v_{o}}, \rho_{2 v_{o}}\right)$ and $N=0$. In the same way when $\rho_{i v}=\rho_{i m}$, then $N=0$. Consequently, the number of drops per unit volume admits a maximum when $\left.\rho_{i v} \in\right] \rho_{i v_{o}}, \rho_{i m}[$. In the case for which $\rho_{1 m}$ and $\rho_{2 m}$ are close from $\rho_{1 v_{o}}, \rho_{2 v_{o}}$, explicit calculations are simplified.

Owing to the fact we can chose the chemical potentials and the free energy as

$\Psi\left(\rho_{1 l_{o}}, \rho_{2 l_{o}}\right)=\Psi\left(\rho_{1 v_{o}}, \rho_{2 v_{o}}\right)=0$

$\Psi_{\rho_{1}}^{\prime}\left(\rho_{1 l_{o}}, \rho_{2 l_{o}}\right)=\Psi_{\rho_{1}}^{\prime}\left(\rho_{1 v_{o}}, \rho_{2 v_{o}}\right)=0$

$\Psi_{\rho_{2}}^{\prime}\left(\rho_{1 l_{o}}, \rho_{2 l_{o}}\right)=\Psi_{\rho_{2}}^{\prime}\left(\rho_{1 v_{o}}, \rho_{2 v_{o}}\right)=0$,

near equilibrium positions, the free energy expansions are

$$
\begin{aligned}
& \Psi\left(\rho_{1 l}, \rho_{2 l}\right)=\frac{1}{2}\left(\rho_{1 l}-\rho_{1 l_{o}}\right)^{2} \Psi_{\rho_{1}^{2}}^{\prime \prime}\left(\rho_{1 l_{o}}, \rho_{2 l_{o}}\right) \\
& \quad+\left(\rho_{1 l}-\rho_{1 l_{o}}\right)\left(\rho_{2 l}-\rho_{2 l_{o}}\right) \Psi_{\rho_{1} \rho_{2}}^{\prime \prime}\left(\rho_{1 l_{o}}, \rho_{2 l_{o}}\right)+\frac{1}{2}\left(\rho_{2 l}-\rho_{2 l_{o}}\right)^{2} \Psi_{\rho_{2}^{2}}^{\prime \prime}\left(\rho_{1 l_{o}}, \rho_{2 l_{o}}\right) \\
& \Psi\left(\rho_{1 v}, \rho_{2 v}\right)=\frac{1}{2}\left(\rho_{1 v}-\rho_{1 v_{o}}\right)^{2} \Psi_{\rho_{1}^{2}}^{\prime \prime}\left(\rho_{1 v_{o}}, \rho_{2 v_{o}}\right) \\
& \quad+\left(\rho_{1 v}-\rho_{1 v_{o}}\right)\left(\rho_{2 v}-\rho_{2 v_{o}}\right) \Psi_{\rho_{1} \rho_{2}}^{\prime \prime}\left(\rho_{1 v_{o}}, \rho_{2 v_{o}}\right)+\frac{1}{2}\left(\rho_{2 v}-\rho_{2 v_{o}}\right)^{2} \Psi_{\rho_{2}^{2}}^{\prime \prime}\left(\rho_{1 v_{o}}, \rho_{2 v_{o}}\right)
\end{aligned}
$$

and the chemical potential expansions are

$$
\begin{aligned}
& \Psi_{\rho_{1}}^{\prime}\left(\rho_{1 v}, \rho_{2 v}\right)=\left(\rho_{1 v}-\rho_{1 v_{o}}\right) \Psi_{\rho_{1}^{2}}^{\prime \prime}\left(\rho_{1 v_{o}}, \rho_{2 v_{o}}\right)+\left(\rho_{2 v}-\rho_{2 v_{o}}\right) \Psi_{\rho_{1} \rho_{2}}^{\prime \prime}\left(\rho_{1 v_{o}}, \rho_{2 v_{o}}\right) \\
& \Psi_{\rho_{1}}^{\prime}\left(\rho_{1 l}, \rho_{2 l}\right)=\left(\rho_{1 l}-\rho_{1 l_{o}}\right) \Psi_{\rho_{1}^{2}}^{\prime \prime}\left(\rho_{1 l_{o}}, \rho_{2 l_{o}}\right)+\left(\rho_{2 l}-\rho_{2 l_{o}}\right) \Psi_{\rho_{1} \rho_{2}}^{\prime \prime}\left(\rho_{1 l_{o}}, \rho_{2 l_{o}}\right) \\
& \Psi_{\rho_{2}}^{\prime}\left(\rho_{1 v}, \rho_{2 v}\right)=\left(\rho_{1 v}-\rho_{1 v_{o}}\right) \Psi_{\rho_{1} \rho_{2}}^{\prime \prime}\left(\rho_{1 v_{o}}, \rho_{2 v_{o}}\right)+\left(\rho_{2 v}-\rho_{2 v_{o}}\right) \Psi_{\rho_{2}^{2}}\left(\rho_{1 v_{o}}, \rho_{2 v_{o}}\right) \\
& \Psi_{\rho_{2}}^{\prime}\left(\rho_{1 l}, \rho_{2 l}\right)=\left(\rho_{1 l}-\rho_{1 l_{o}}\right) \Psi_{\rho_{1} \rho_{2}}^{\prime \prime}\left(\rho_{1 l_{o}}, \rho_{2 l_{o}}\right)+\left(\rho_{2 l}-\rho_{2 l_{o}}\right) \Psi_{\rho_{2}^{2}}^{\prime \prime}\left(\rho_{1 l_{o}}, \rho_{2 l_{o}}\right)
\end{aligned}
$$

From the equality of chemical potentials in the two bulks $l$ and $v$, one obtains

$2\left(p_{l}-p_{v}\right)=\left(\rho_{1 l}+\rho_{1 l_{o}}-\rho_{1 v}-\rho_{1 v_{o}}\right) \psi_{1}^{\prime}(v)+\left(\rho_{2 l}+\rho_{2 l_{o}}-\rho_{2 v}-\rho_{2 v_{o}}\right) \psi_{2}^{\prime}(v)$

and from relations (11),

$$
\begin{aligned}
2\left(p_{l}-p_{v}\right)= & \left(\rho_{1 v}-\rho_{1 v_{o}}\right)\left(\rho_{1 l}+\rho_{1 l_{o}}-\rho_{1 v}-\rho_{1 v_{o}}\right) \Psi_{\rho_{1}^{2}}^{\prime \prime}\left(\rho_{1 v_{o}}, \rho_{2 v_{o}}\right) \\
+ & \left(\rho_{2 v}-\rho_{2 v_{o}}\right)\left(\rho_{1 l}+\rho_{1 l_{o}}-\rho_{1 v}-\rho_{1 v_{o}}\right) \Psi_{\rho_{1} \rho_{2}}^{\prime \prime}\left(\rho_{1 v_{o}}, \rho_{2 v_{o}}\right) \\
+ & \left(\rho_{1 v}-\rho_{1 v_{o}}\right)\left(\rho_{2 l}+\rho_{2 l_{o}}-\rho_{2 v}-\rho_{2 v_{o}}\right) \Psi_{\rho_{1}}^{\prime \prime}\left(\rho_{2}\left(\rho_{1 v_{o}}, \rho_{2 v_{o}}\right)\right. \\
+ & \left(\rho_{2 v}-\rho_{2 v_{o}}\right)\left(\rho_{2 l}+\rho_{2 l_{o}}-\rho_{2 v}-\rho_{2 v_{o}}\right) \Psi_{\rho_{2}^{\prime \prime}}^{\prime \prime}\left(\rho_{1 v_{o}}, \rho_{2 v_{o}}\right)
\end{aligned}
$$


Vapor and gas constitute a perfect gas mixture and the volume free energy is the sum of the volume free energies of each fluid In bulk $v$,

$\Psi\left(\rho_{1}, \rho_{2}\right)=\Psi_{1}\left(\rho_{1}\right)+\Psi_{2}\left(\rho_{2}\right)$

and consequently,

$$
\begin{aligned}
p_{l}-p_{v}=\frac{1}{2}( & \left.\rho_{1 v}-\rho_{1 v_{o}}\right)\left(\rho_{1 l}+\rho_{1 l_{o}}-\rho_{1 v}-\rho_{1 v_{o}}\right) \Psi_{1}^{\prime \prime}\left(\rho_{1 v_{o}}\right) \\
& +\frac{1}{2}\left(\rho_{2 v}-\rho_{2 v_{o}}\right)\left(\rho_{2 l}+\rho_{2 l_{o}}-\rho_{2 v}-\rho_{2 v_{o}}\right) \Psi_{2}^{\prime \prime}\left(\rho_{2 v_{o}}\right)
\end{aligned}
$$

where $p_{i}\left(\rho_{i}\right)=\rho_{i} \Psi_{i}^{\prime}\left(\rho_{i}\right)-\Psi_{i}\left(\rho_{i}\right)+p_{i_{o}}$, with $p_{i_{o}}$ as an additive constant, denotes the partial pressure of the component $i$ of the mixture in the bulk $v$ and $p\left(\rho_{1}, \rho_{2}\right)=p_{1}\left(\rho_{1}\right)+p_{2}\left(\rho_{2}\right)$. Let us notice that $\rho_{1 l}$ is the liquid density when $\rho_{1 v}, \rho_{2 l}, \rho_{2 v}$ denote the gas densities. Moreover $\rho_{1 v_{o}} \Psi_{1}^{\prime \prime}\left(\rho_{1 v_{o}}\right)=\frac{\partial p_{1}}{\partial \rho_{1}}\left(\rho_{1 v_{o}}\right)=c_{1}^{2}$ where $c_{1}$ is the isothermal celerity of the saturated vapor at the liquid-vapor equilibrium. With component molar masses of the same order, $c_{1}$ and the isothermal celerity of sound in the gas are of the same magnitude. The second term of relation (12) is negligible with respect to the first one and we obtain the simple result

$p_{l}-p_{v} \approx\left(\rho_{1 v}-\rho_{1 v_{o}}\right) \rho_{1 l_{o}} \Psi_{1}^{\prime \prime}\left(\rho_{1 v_{o}}\right)$

Relation (10) yields

$N=K \frac{\rho_{1 m}-\rho_{1 v}}{\rho_{1 l}-\rho_{1 v}}\left(\rho_{1 v}-\rho_{1 v_{o}}\right)^{3} \rho_{1 l_{o}}^{3}\left[\Psi_{1}^{\prime \prime}\left(\rho_{1 v_{o}}\right)\right]^{3}$

By noticing that $\rho_{1 l}-\rho_{1 v} \approx \rho_{1 l_{o}}$, one obtains

$N \approx K\left(\rho_{1 m}-\rho_{1 v}\right)\left(\rho_{1 v}-\rho_{1 v_{o}}\right)^{3} \rho_{1 l_{o}}^{2}\left[\Psi_{1}^{\prime \prime}\left(\rho_{1 v_{o}}\right)\right]^{3}$

expression which is maximum when $\rho_{1 v}=\frac{3 \rho_{1 m}+\rho_{1 v_{0}}}{4}$.

One deduces the maximum value of $N$

$N_{\max }=\frac{1}{32 \pi \sigma^{3}}\left(\rho_{1 v}-\rho_{1 v_{o}}\right)^{4} \rho_{1 l_{o}}^{2}\left[\Psi_{1}^{\prime \prime}\left(\rho_{1 v_{o}}\right)\right]^{3}$

Let us notice that $\Psi_{1}\left(\rho_{1 v}\right)-\Psi_{1}\left(\rho_{1 v_{o}}\right)=\frac{1}{2}\left(\rho_{1 v}-\rho_{1 v_{o}}\right)^{2} \Psi_{1}^{\prime \prime}\left(\rho_{1 v_{o}}\right)$ and by taking into account the choice of the chemical potential components,

$\rho_{1 v_{o}} \Psi_{1}^{\prime}\left(\rho_{1 v_{o}}\right)-\rho_{1 v} \Psi_{1}^{\prime}\left(\rho_{1 v}\right)=\rho_{1 v_{o}}\left[\Psi_{1}^{\prime}\left(\rho_{1 v_{o}}\right)-\Psi_{1}^{\prime}\left(\rho_{1 v}\right)\right]+\left(\rho_{1 v_{o}}-\rho_{1 v}\right) \Psi_{1}^{\prime}\left(\rho_{1 v}\right)=\rho_{1 v_{o}}\left(\rho_{1 v_{o}}-\right.$ $\left.\rho_{1 v}\right) \Psi_{1}^{\prime \prime}\left(\rho_{1 v_{o}}\right)-\left(\rho_{1 v_{o}}-\rho_{1 v}\right)^{2} \Psi_{1}^{\prime \prime}\left(\rho_{1 v_{o}}\right)$.

Consequently, $p_{1 v_{o}}-p_{1 v}=\frac{1}{2}\left(\rho_{1 v_{o}}-\rho_{1 v}\right)\left(\rho_{1 v_{o}}+\rho_{1 v}\right) \Psi_{1}^{\prime \prime}\left(\rho_{1 v_{o}}\right) \approx \rho_{1 v_{o}}\left(\rho_{1 v_{o}}-\rho_{1 v}\right) \Psi_{1}^{\prime \prime}\left(\rho_{1 v_{o}}\right)$, and relation (15) yields

$N_{\max }=\frac{\rho_{1 l_{o}}^{2}\left[p_{1 v}-p_{1 v_{o}}\right]^{4}}{32 \pi \sigma^{3} \rho_{1 v_{o}}^{3} c_{1}^{2}}$

where $p_{1 v}, p_{1 v_{o}}$ denote the partial pressures of the supersaturated vapor and the saturated vapor. 
When $p_{v}=p_{1 v}+p_{2}$ where $p_{2}$ denotes the partial pressure of the gas assumed to be independent of the quantity of vapor of the component 1 , one obtains

$N_{\max }=\frac{\rho_{1 l_{o}}^{2}\left[p_{v}-p_{v_{o}}\right]^{4}}{32 \pi \sigma^{3} \rho_{1 v_{o}}^{3} c_{1}^{2}}$

This case corresponds to a vapor density smaller than the gas density. It is generally the case for water vapor in air at ambient temperature.

For example, we consider the physical values of liquid water at the temperature of $25^{\circ} \mathrm{C}$ : surface tension $\sigma=72$ dyne $/ \mathrm{cm}$, isothermal sound velocity $c_{1}=2.810^{4} \mathrm{~cm} / \mathrm{s}, \rho_{1 l_{o}}=1$ $\mathrm{g} / \mathrm{cm}^{3}, \rho_{1 v_{o}}=2.310^{-5} \mathrm{~g} / \mathrm{cm}^{3}$ and for $\frac{p_{1 v}-p_{1 v_{o}}}{p_{1 v_{o}}}=510^{-5}$ corresponding to a water vapor supersaturated of 0.2 per 100 , one obtains a maximum of droplets $N_{\text {max }}=15000$ per cubic centimeter of gas [6].

\section{ENERGY OF FORMATION OF DROPLETS}

From relations (7) and (9), the capillary energy of formation of $n$ droplets is given by the expression

$E_{c}=\frac{3}{2} \frac{n}{K^{\frac{1}{3}}} v_{l}^{\frac{2}{3}} \equiv \frac{3}{2} \frac{\rho_{1 m}-\rho_{1 v}}{\rho_{1 l}-\rho_{1 v}} V\left(p_{l}-p_{v}\right)$

According to relation (10) one obtains,

$E_{c} \approx \frac{3}{2}\left(\rho_{1 m}-\rho_{1 v}\right)\left(\rho_{1 v}-\rho_{1 v_{o}}\right) \Psi_{1}^{\prime \prime}\left(\rho_{1 v_{o}}\right) V$

From the choice of the mixture free energy, the total free energy of formation of the droplets is $E=E_{0}+E_{c}$ where $E_{0}$ is the bulk free energy. One deduces

$E_{o}=n \psi(l) v_{l}+\psi(v) v_{v}=\frac{V}{\rho_{1 l}-\rho_{1 v}}\left[\left(\rho_{1 m}-\rho_{1 v}\right) \psi(l)+\left(\rho_{1 l}-\rho_{1 m}\right) \psi(v)\right]$

By taking into account the equality of the component chemical potentials (11) and due to the fact that gas and vapor are perfect gases, $\Psi_{\rho_{1} \rho_{2}}^{\prime \prime}=0$, one obtains

$$
\begin{aligned}
& 2\left(\rho_{1 l}-\rho_{1 v}\right) e_{o}= \\
& \left(\rho_{1 m}-\rho_{1 v}\right)\left[\left(\rho_{1 l}-\rho_{1 l_{o}}\right)\left(\rho_{1 v}-\rho_{1 v_{o}}\right) \Psi_{1}^{\prime \prime}\left(\rho_{1 v_{o}}\right)+\left(\rho_{2 l}-\rho_{2 l_{o}}\right)\left(\rho_{2 v}-\rho_{2 v_{o}}\right) \Psi_{2}^{\prime \prime}\left(\rho_{2 v_{o}}\right)\right] \\
& +\left(\rho_{1 l}-\rho_{1 m}\right)\left[\left(\rho_{1 v}-\rho_{1 v_{o}}\right)^{2} \Psi_{1}^{\prime \prime}\left(\rho_{1 v_{o}}\right)+\left(\rho_{2 v}-\rho_{2 v_{o}}\right)^{2} \Psi_{2}^{\prime \prime}\left(\rho_{2 v_{o}}\right)\right]
\end{aligned}
$$

with $e_{o}=\frac{E_{o}}{V}$. Due to the respective order of $\rho_{1 l}, \rho_{1 v}, \rho_{2 l}, \rho_{2 v}$, we deduce

$e_{o} \approx \frac{1}{2}\left[\left(\rho_{1 v}-\rho_{1 v_{o}}\right)^{2} \Psi_{1}^{\prime \prime}\left(\rho_{1 v_{o}}\right)+\left(\rho_{2 v}-\rho_{2 v_{o}}\right)^{2} \Psi_{2}^{\prime \prime}\left(\rho_{2 v_{o}}\right)\right]$

As indicated in paragraph $3, \rho_{1 v o} \Psi_{1}^{\prime \prime}\left(\rho_{1 v_{o}}\right)$ and $\rho_{2 v o} \Psi_{2}^{\prime \prime}\left(\rho_{2 v_{o}}\right)$ are of same order. Under usual thermodynamic conditions, the vapor density is much lower than the gas density; 
moreover $\rho_{2 v}-\rho_{2 v_{o}}$ associated with a small variation of gas density is of the same order (or an order lower) than $\rho_{1 v}-\rho_{1 v_{o}}$ associated with variation of vapor density. Then,

$e_{o} \approx \frac{1}{2}\left(\rho_{1 v}-\rho_{1 v_{o}}\right)^{2} \Psi_{1}^{\prime \prime}\left(\rho_{1 v_{o}}\right)$

and

$e \approx \frac{1}{2}\left[\left(\rho_{1 v}-\rho_{1 v_{o}}\right)^{2}+3\left(\rho_{1 m}-\rho_{1 v}\right)\left(\rho_{1 v}-\rho_{1 v_{o}}\right)\right] \Psi_{1}^{\prime \prime}\left(\rho_{1 v_{o}}\right)$

is the formation free energy of droplets per unit volume.

This energy has a maximum value when the number of drops per unit volume is maximum. The value of this energy is

$e_{\max }=\left(\rho_{1 v}-\rho_{1 v_{o}}\right)^{2} \Psi_{1}^{\prime \prime}\left(\rho_{1 v_{o}}\right)$

and by a calculus as in paragraph 3 ,

$e_{\max } \approx \frac{\left[p_{1 v}-p_{1 v_{o}}\right]^{2}}{\rho_{1 v o} c_{1}^{2}}$

When $p_{v}=p_{1 v}+p_{2}$, in the same way that in paragraph 3 ,

$e_{\max } \approx \frac{\left[p_{v}-p_{v_{o}}\right]^{2}}{\rho_{1 v o} c_{1}^{2}}$

This value does not depend on the surface tension.

\section{CONCLUSION}

The approach to determine the droplet number takes into account the fact that the mixture free energy is a non-convex function of the densities of components. However, this result is not used in explicit calculations and the maximum number of droplets does not take into account the non-convex part of the free energy since the densities of components are near equilibrium densities associated with the liquid and its saturated vapor in presence of a gas. The result is obtained for a gas and a vapor considered as perfect gases. It cannot be extended to micro-bubbles in a liquid because the free energy of the liquid phase is not an additive function of the mixture components.

\section{REFERENCES}

1. Y.S. Djikaev and J. Teichmann, J. Aerosol Sci. 30 (1999) 587.

2. V.V. Levdansky et al, Int. J. Heat and Mass Transfert. 45 (2002) 3831.

3. H. Gouin, Eur. J. Mech./B Fluids. 9 (1990) 469.

4. Y. Rocard, Thermodynamique, Masson, Paris, 1952.

5. J.S. Rowlinson and B. Widom, Molecular theory of capillarity, Clarendon Press, Oxford, 1984.

6. R.C. Weast, Handbook of Chemistry and Physics, 65th edition, CRC Press, Boca Raton, Florida, 1992. 